\title{
Wurst-Mediated Airway Clearance is Required for Postembryonic Development
}

Anna Lollies, Tamara Krsmanovic, Laura CA Jussen and Matthias Behr*

Life \& Medical Sciences Institute (LIMES), Laboratory for Molecular Developmental Biology, University of Bonn, Carl-Troll-Strasse 31, 53115 Bonn, Germany

\begin{abstract}
Tubular organs are required for transport of liquids or gases. The Drosophila tracheal system and the human lungs need to undergo liquid to air transition to enable oxygen transport in the postembryonic or neonatal development respectively. In humans, a failure of airway liquid-clearance can lead to severe clinical syndromes, such as neonatal respiratory distress syndrome or transient tachypnea of newborn. However, the underlying molecular mechanisms are poorly understood. Previously, we identified the transmembrane J-domain protein Wurst as a key regulator of airway clearance in Drosophila. Wurst is involved in endocytosis and recruits clathrin and the associated Heat shock cognate protein 70 to the apical membrane. In wurst mutant embryos airways are oversized and airway liquidclearance is impaired. Here we show that wurst expression in tracheal cells, but not in the respiratory openings, is sufficient to rescue airway clearance and air-filling. We found that only tracheal specific RNAi knock-down of wurst is essential for airway liquid-clearance in post embryonic stages resulting in severe growth arrest and early lethality. In the tracheal wurst knock-down, clathrin accumulated at the apical cell membrane. Cell culture assays identified Wurst co-localization with endocytic markers and co-clustering at the plasma membrane. In summary, our data characterize Wurst as a key regulator of airway liquid-clearance and physiology. Wurst is conserved and a single ortholog was identified in humans, termed DNAJC22. Thus, investigating the molecular function of Wurst/DNAJC22 in airway liquid-clearance will contribute to a better understanding of clinical relevant lung syndromes.
\end{abstract}

Keywords: Airways; Endocytosis; Extracellular matrix; Liquidclearance; Respiratory distress; Tracheal system; Wurst/DNAJC22

\section{Introduction}

Lungs, the cardio-vascular system, kidneys and many other tube organs transport gases or fluids through a network of ramified tubular branches or vessels. Morphogenesis of branching and patterning of the respiratory tubes involves a number of genetic pathways [1]. Key signaling pathways and molecular mechanisms are evolutionarily conserved and are required for the development of respiratory systems among higher organisms $[2,3]$. The developing human lungs are net secreting organs where fluids in the tube lumina control organ size $[4,5]$. Before birth, the lung epithelium switches to net absorption, resulting in a fast process of airway liquid-clearance, which is essential for respiration of newborns. Defects in airway liquid-clearance have the potential to cause severe clinical syndromes, such as transient tachypnea of newborn (TTN) and the neonatal respiratory distress syndrome (nRDS) [4,5]. Furthermore, it has been discussed that edema liquid-clearance may require similar molecular mechanisms. However, little is known about molecular mechanisms underlying airway and edema liquid-clearance.

Drosophila melanogaster represents an ideal model organism for studying genetic and molecular mechanisms of a number of human diseases such as Alzheimer, diabetes and cancer [6,7]. The advantages of fast forward genetics, spatial and temporal specific over expression studies and a short generation time of the fly represent optimal preconditions to analyze conserved mechanisms and pathways involved in airway physiology. The Drosophila tracheal system serves as a prototypical model of genetically ordered branching [2,8]. It arises from epidermal cell clusters that develop a highly ordered stereotyped pattern of tubular branches during the second half of embryogenesis. Several conserved signaling pathways, like Fibroblast growth factor (FGF), Epidermal growth factor (EGF), wingless (wnt) and Notch signaling control tracheal branching [8-10]. In addition, extracellular matrix (ECM) components are secreted into the forming tube lumina. ECM components form a transient luminal cylinder in order to limit the tube size and to coordinate uniform tube lumen diameter expansion $[10,11]$. At the end of embryogenesis, Drosophila airways need to clear the luminal ECM cylinder. In addition, airway liquid-clearance enables accompanied air-filling $[11,12]$. Previously we showed that Wurst is required for embryonic airway protein and liquid clearance $[13,14]$. Wurst encodes for a transmembrane protein which contains a wellconserved DnaJ-domain and a clathrin binding motif at the C-terminus [13]. Single orthologs with a similar domain structure have been identified in vertebrates, referred to as DNAJC22 (DNAJC-domain at the C-terminus, number 22; [H. sapiens-NP_079178.2; P. troglodytesXP_001158376.1; C. lupus-XP_543683.2; B. teurus-NP_001069169.1; M. musculus-NP_789805.1; $R$. norvegicus-NP_001014226.1; D. rerioXP_001335380.1]) [15]. Drosophila Wurst interacts with clathrin and the clathrin coat component Heat shock cognate protein (Hsc) 70-4 and recruits both to the apical cell membrane $[13,14]$. RNAi-mediated Wurst knock-down resulted in inhibition of endocytosis in Drosophila Schneider (SL2) cells [13]. Genetic studies in epidermal tissues revealed a mutual dependency of Wurst and Clathrin heavy chain (Chc) [13], which is a key component of the clathrin coat [16]. Here we investigate

*Corresponding author: Matthias Behr, Life \& Medical Sciences Institute (LIMES) Laboratory for Molecular Developmental Biology, University of Bonn, Carl-TrollStrasse 31, 53115 Bonn, Germany, Tel: +49 228 7362746; Fax: +49 2287362643 E-mail:mbehr@uni-bonn.de

Received November 22, 2011; Accepted January 21, 2012; Published January 25, 2012

Citation: Lollies A, Krsmanovic T, Jussen LCA, Behr M (2012) Wurst-Mediated Airway Clearance is Required for Postembryonic Development. J Aller Ther S7:002. doi:10.4172/2155-6121.S7-002

Copyright: @ 2012 Lollies A, et al. This is an open-access article distributed unde the terms of the Creative Commons Attribution License, which permits unrestricted use, distribution, and reproduction in any medium, provided the original author and source are credited. 
the role of Wurst in tracheal cells as a key modulator of airway liquidclearance and its importance for postembryonic development.

\section{Materials and Methods}

Immunofluorescence studies, embryo fixation and air-filling assay

Embryos were dechorionated with $2.5 \%$ sodium hypochlorite (5 $\mathrm{min}$ ) and fixed in $2 \mathrm{ml} 4 \%$ PFA (paraformaldehyde) and $3 \mathrm{ml}$ heptane for 20 minutes. Afterwards they were devitellinized ( $3 \mathrm{ml}$ heptane and $10 \mathrm{ml}$ methanol). Fluorescence immunolabeling and the RNA in situ hybridization studies were performed as described in [17]. The fluorescein-conjugated Cbp (1:100, New England Biolabs) is a recombinant fusion protein that binds specifically to chitin. We used the following antibodies: anti-clathrin (goat, 1:50; Sigma, [13]); anti-Chc (rat, 1:40 [17]); anti-Hsc70 (mouse, 1:100, Stressgene [13]); anti-RFP (rabbit, 1:50); anti-Wurst C (guinea pig, 1:40 [13,14]). For co-localization assays with anti-Chc a new anti-Wurst $\mathrm{N}$ antibody was generated (guinea pig) recognizing the $\mathrm{N}$-terminus [GKDKTNDTSKGSPSKAKSNG]. After final bleeding, the anti-Wurst antibody was affinity-purified by using the $\mathrm{N}$-terminal peptide and tested in wild-type and in Wurst over expression embryos (not shown) and by peptide competition (Supplementary Figure S1). The anti-Wurst $\mathrm{N}$ and the anti-Wurst $\mathrm{C}$ [13] antibodies detect an identical expression pattern in wild-type embryos. UAS-GAL4 mediated over expression of Wurst was detected by the anti-Wurst $\mathrm{N}$ antibody. In summary, our data show, that the antibody recognizes the $\mathrm{N}$-terminus of the Wurst protein. The immune fluorescence studies were performed with a Zeiss Axiovert (SL2 cells and membrane sheets). Confocal images were achieved by using a Zeiss LSM710. Each fluorochrome was scanned in single optical sections. For confocal microscopy Pinhole "airy 1"
Zeiss standard settings were employed using a Zeiss 63x Pan Neofluar objective. Images were analyzed using Axiovision and ZEN software and cut in Photoshop CS5. Figures were prepared with Illustrator CS5. For analyzing air-filling, dechorionated embryos at stage 17 were monitored by bright-field microscopy using a Zeiss Axiovert and the Axiovision software. The detailed procedure is described in [14,17].

\section{Generation of the UAS-Wurst-red fluorescent protein ( $r f p)$ fusion construct}

A UAS-Wurst-rfp transgene was generated as follows. The Wurst open reading frame was amplified by PCR using the primer pair [forward primer: GAATTCGCCTCTCTTTCTCCAATTCTTCGC; reverse primer: GGTACCTTAAGGACGACGATGGCCTCCTCCTC] and the cDNA LD21896.The PCR product was digested with EcoRI and $\mathrm{KpnI}$ and cloned in frame in front of an $r f p$ open reading frame. PCR products were confirmed by sequencing. The resulting Wurst$r f p$ cassette was then digested with EcoRI and NotI before cloning it into the pUAST vector. Transgenic lines were established by germ line transformation and tested in vivo for RFP expression and for Wurst expression by immune fluorescent labeling studies using the antiWurst antibodies.

\section{Fly stocks, rescue experiment and cell culture}

Fly stocks were obtained from the Bloomington (http://flystocks. bio.indiana.edu/) and Vienna (www.vdrc.at) stock centers: $\mathrm{w}^{1118}$ (referred to as wild-type), breathless GAL4, decapentaplegic GAL4, paired GAL4; UAS-WurstRNAi $i^{\# 110270}$ [14]. Wurst $t^{l(1) G 0162}$ is described in [13]. The Wurst gene locus is located at the first (X)-chromosome. The Wurst ${ }^{t(1) G 0162} /$ Ymales are embryonic lethal and show impaired airfilling. For the rescue experiment, Wurst heterozygous females which

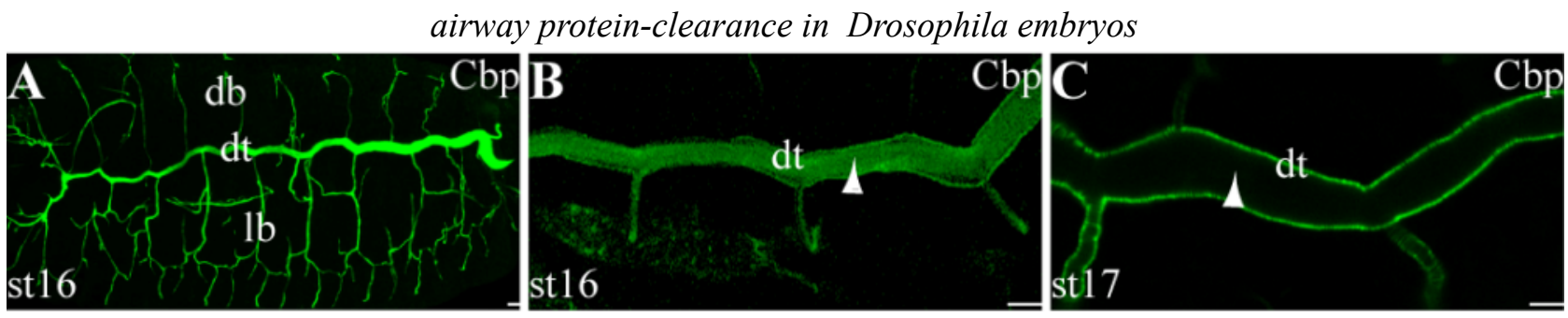

airway liquid clearance and air-filling
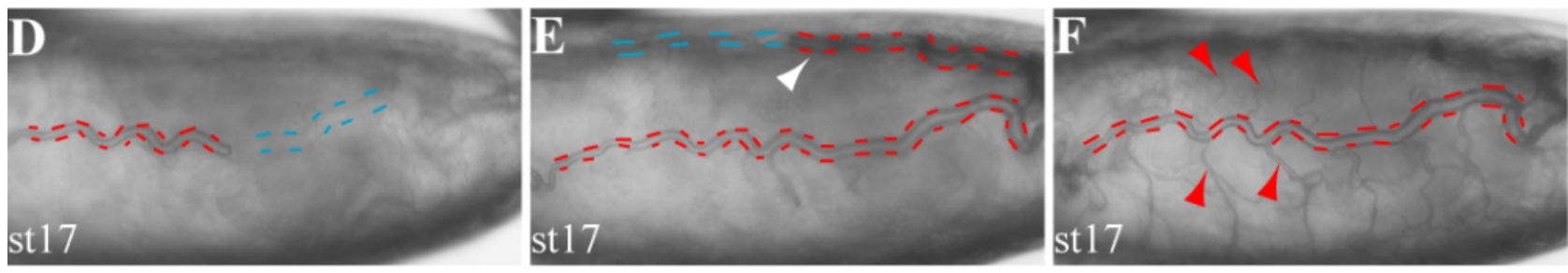

Figure 1: Airway clearance in Drosophila embryos

Confocal images (A-C) show whole mount (A) and magnifications of the trachea $(B, C)$ at stage 16 and stage 17 embryos. For detecting tracheal tube lumina the fluorescein conjugated chitin-binding-probe ( $\mathrm{Cbp}$ ) was used. Cbp recognizes chitin. (A) Three-dimensional projection of confocal Z-stacks across the tracheal system visualizes chitinous tracheal tubes. The main airways are indicated as dorsal trunk (dt), dorsal branches (db), lateral branches (lb). (B) In stage 16 embryos single confocal sections show that chitin is localized in a transient luminal cylinder-like matrix (arrowheads), which controls tube expansion. (C) At the end of embryogenesis, at stage 17, airway lumina (arrowhead) are cleared from the chitin cylinder. Scale bars represent $10 \mu \mathrm{m}$. (D-F) At stage 17, embryos undergo airway liquid-clearance and air-filling. This process can be detected due to different light diffractions by bright-field microscopy. Red dashes indicate air-filled airways, blue dashes liquid-filled lumina. The air-filling process starts in the mid of one dorsal trunk $(D)$, expands along the main airway including also the other hemisphere ( $E$, white arrowhead) until all airways are aerated $(F)$. Red arrowheads point to small dorsal and lateral tracheal tubes. All images show anterior to the left and dorsal to the top. 
contain a UAS-wurst-rfp construct at the second chromosome were mated with males bearing the desired GAL4 driver construct. In the progeny Wurst $t^{(1) G 0162} /$ Ymales were tested for Wurst-RFP expression and air-filling. For all experiments balancer strains (FM7) with GFP were used to identify individual genotypes.

For cell culture experiments Drosophila SL2 cells were weekly subcultured $\left(3 \times 10^{6}\right.$ cells $\left./ \mathrm{ml}\right)$ at $25^{\circ} \mathrm{C}$ in Drosophila Schneider Medium (Invitrogen), 10\% fetal bovine serum (heat inactivated) and $1 \%$ penicillin/streptomycin (Sigma). For generating SL2 membrane sheets cells were grown on a Poly-L-lysine coated cover slip. For sonication, a petri dish was used as sonication chamber containing ice-cold sonication buffer. A sonication pulse was applied for $1 \mathrm{sec}$. The resulting membrane sheets were analyzed with TMA-DPH, a phospholipid membrane marker, and by fluorescence microscopy and were afterwards fixed in PFA. For immunofluorescent labeling studies
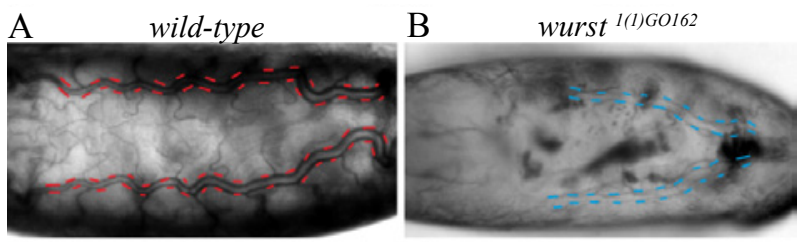

C wild-type

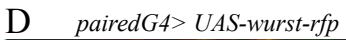
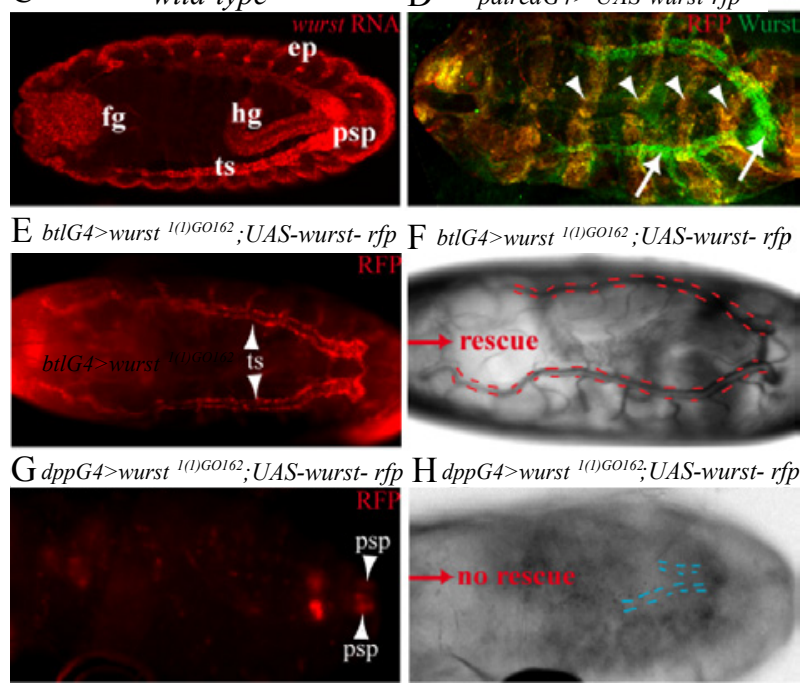

Figure 2: Wurst-mediated airway clearance is tracheal specific and independent of posterior spiracles

Bright-field in vivo microscopy $(\mathrm{A}, \mathrm{B}, \mathrm{F}, \mathrm{H})$ of late embryos at stage 17 . Red dashes indicate air-filled, blue dashes liquid-filled airways. Confocal $(C, D)$ and in vivo fluorescence microscopy $(E, G)$ images of stage 16 embryos. (A) Wild-type embryos undergo air-filling (B) wurst(1)G0162 mutant embryos lack air-filling (C) RNA in situ hybridization reveals wurst transcript expression in the foregut (fg) hindgut (hg), epidermis (ep), tracheal system (ts) and posterior spiracles (psp). (D) Wurst and RFP were specifically expressed under the control of the UASGAL4 system. Immunofluorescence studies using anti-Wurst and anti-RFP antibodies reveal ectopic Wurst and RFP co-expression (orange, arrowheads) in the characteristic stripe pattern of the paired GAL4 driver line. Note the additional endogenous Wurst (green) expression in tracheal cells and posterio spiracles (arrows). (E,F) The failure of air-filling in wurstt(1)G0162 mutants can be rescued by ectopically expressing wurst in tracheal cells, using the tracheal specific bt/G4 driver line. Arrowheads in E point to RFP expression in the tracheal system (ts). (G,H) The failure of air-filling in wurst(1)G0162 mutants canno be rescued by ectopically expressing wurst in posterior spiracle (psp) cells, using the $d p p \mathrm{G} 4$ driver line. Arrowheads in $\mathrm{G}$ point to the RFP expression in $\mathrm{psp}$.
anti-Wurst C [13] was combined with aniti-clathrin or anti-Hsc70 antibodies and anti-Wurst $\mathrm{N}$ was combined with anti-Chc antibody.

\section{Results}

\section{Wurst, a key regulator of airway clearance and air-filling}

The tracheal tube lumina of Drosophila wild-type embryos contain a cylindrical extracellular chitinous matrix (Figure 1A,B). At the end of embryogenesis (stage 17), the chitinous-ECM and liquids are cleared from the tube lumina (Figure 1C) [13], enabling gas filling. The liquidclearance and accompanied air-filling process can be monitored in vivo by bright-field microscopy due to differences in light diffraction caused by transition from liquid- to gas-filled airways [14]. During aeration, air expands from the mid of the dorsal main airway along the dorsal most posterior branch, enters the main airway of the other hemisphere and then extends into smaller airways (Figure 1D-F). Shortly after airfilling is completed, the larva hatches and conducts oxygen between the respiratory openings, the posterior spiracles and internal tissues. In a previous screen for regulators of airway physiology, we found that Wurst ${ }^{(1) G 0162}$ (transposable P-element induced) [13] mutant embryos lack air-filling, when compared to wild-type embryos (Figure 2A,B) $[13,14]$.

We tested the Wurst gene expression by antisense RNA hybridization. The Wurst transcript was strongly expressed in tracheal cells as well as in the posterior spiracles (Figure 2C) which are the only openings that connect the tracheal branches with the environment for gas exchange during early stages of post embryonic development. The Wurst $t^{(1) G 0162}$ mutation affects all Wurst expressing organs, including trachea and posterior spiracles. In order to determine the importance of Wurst for airway clearance, we addressed the question whether organ specific Wurst expression is able to rescue Wurst ${ }^{l(1) G 0162}$ mutant air-filling defects. In Drosophila, the binary UAS-GAL4 system [18] offers ectopic gene expression in a spatial and temporal manner. In order to perform rescue experiments, we generated a transgenic fly line bearing a UAS-Wurst-rfp (red fluorescence protein) transgene. The expression of the transgene was tested in vivo by mating UAS-Wurst$r f p$ flies with transgenic paired GAL4 flies, which express GAL4 in a characteristic stripe-like pattern. Immunofluorescent confocal analysis of the offspring embryos demonstrated the efficiency of the ectopic Wurst and RFP expression in the expected paired pattern (Figure 2D). The driver line breathless GAL4 (btlG4) was sufficient to express the Wurst-rfp transgene specifically in all tracheal cells. Those embryos did not show obvious defects in tracheal morphology or air-filling (not shown). Next, we ectopically expressed the Wurst-rfp transgene with btlG4 in tracheal cells of Wurst ${ }^{l(1) G 0162}$ mutant embryos (Figure 2E). Strikingly, the resulting Wurst-RFP expression converted tracheal Wurst $t^{(1) G 0162}$ mutant defects into wild-type-like air-filling (Figure 2F). In contrast, ectopic Wurst-RFP expression in cells of posterior spiracle in the Wurst $t^{l(1) G 0162}$ mutants did not rescue air-filling defects (Figure $2 \mathrm{G}, \mathrm{H})$. This demonstrates that only tracheal specific Wurst expression was able to rescue the airway liquid-clearance and air-filling defects in the Wurst ${ }^{\dagger(1) G 0162}$ mutants.

Next, we tested organ specific Wurst knock-down for liquidclearance and air-filling defects. We combined the UAS-GAL4 system with RNAi (interference) technology. For tracheal specific knockdown, we mated $b t l \mathrm{G} 4$ flies with flies bearing a UAS-Wurst-RNAi transgene. In line with recent data [14], the offspring embryos showed strong Wurst reduction in tracheal cells and revealed severe air-filling defects when compared to wild-type (Figure 3A,B). Next we generated RNAi-mediated Wurst knock-down in cells of the posterior spiracle 
using the organ specific driver line decapentaplegic GAL4 (dppG4) [19]. These embryos showed wild-type like air-filling (Figure 3C). In summary, we characterized Wurst as key regulator of tracheal airway physiology in Drosophila. Our findings show the importance of Wurst expression for airway liquid-clearance and air-filling. Wurst controlled liquid-clearance is restricted to tracheal cells, while its role in the posterior spiracles as respiratory openings remains elusive.

\section{Wurst coordinated airway clearance is essential for growth control and survival}

Wild-type embryos and larvae usually survive small periods of hypoxia by rapid and reversible responses [20]. In order to assess the importance of Wurst-mediated airway clearance for tracheal function as an oxygen delivery network, we monitored tracheal specific RNAi Wurst knock-down animals throughout development. Wild-type and animals with Wurst knock-down in posterior spiracles develop normally and finally hatch as adult flies (Figure 3D). In contrast, all tracheal specific RNAi-mediated Wurst knock-down animals died as larvae, in most cases even within the first larval stage (48 hours after egg laying [AEL], Figure 3D). These findings show that Wurst function in tracheal cells, enabling larval respiration, is essential for postembryonic development.

Hypoxic conditions have effects on larval mass gain and trigger the arrest of the developmental progress $[20,21]$. Next, we tested whether failure of air-filling in the tracheal Wurst knock-down mutants has any impact on larval growth and body size control (Figure 3E,F). We monitored freshly hatched wild-type and tracheal specific RNAimediated Wurst knock-down animals throughout larval development.
The wild-type animals dramatically increased their body size and showed darkened, strongly evaginated posterior spiracles until they reached the final larval stage (96 hours AEL; Figure 3E). The Wurst knock-down larvae showed no air-filling but crawled and ingested food like control animals (not shown). Although we observed food uptake, tracheal Wurst knock-down mutants failed to expand their body size. In addition, morphology and brightness of the posterior spiracles indicate the developmental arrest of Wurst knock-down mutants in early larval stages (Figure 3F). In summary, our findings show that embryonic airway liquid-clearance and air-filling is essential for postembryonic development. The inhibition of larvae respiration in Wurst knock down animals causes developmental arrest and early lethality.

\section{Wurst is required for clathrin-mediated endocytosis}

Analysis of the transcript (Figure 2C) identified a broad Wurst expression pattern in epithelial organs which is similar to the recently identified expression pattern of Chc [17]. In co-labeling studies using specific antibodies, we found a strong overlap of Wurst with Clathrin and Hsc70 in Drosophila SL2 cells (Figure 4A,B). In order to analyze Wurst localization in the plasma membrane, we treated the SL2 cells by a previously described method [22], where cells can be grown on cover slips and subjected to brief sonication. This produces layers of plasma membranes, so called membrane sheets, which are devoid of cell constituents $[22,23]$. Immunofluorescent studies showed that Wurst appears small clusters in distinct regions of the SL2 membrane sheets (Figure 4C). Co-labeling studies identified overlap of Wurst clusters with Chc (Figure 4D). Next, we studied Wurst function in clathrinmediated endocytosis in Drosophila embryos. In wild-type tracheal cells

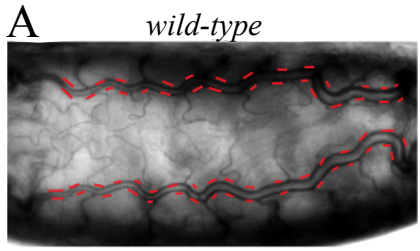

D
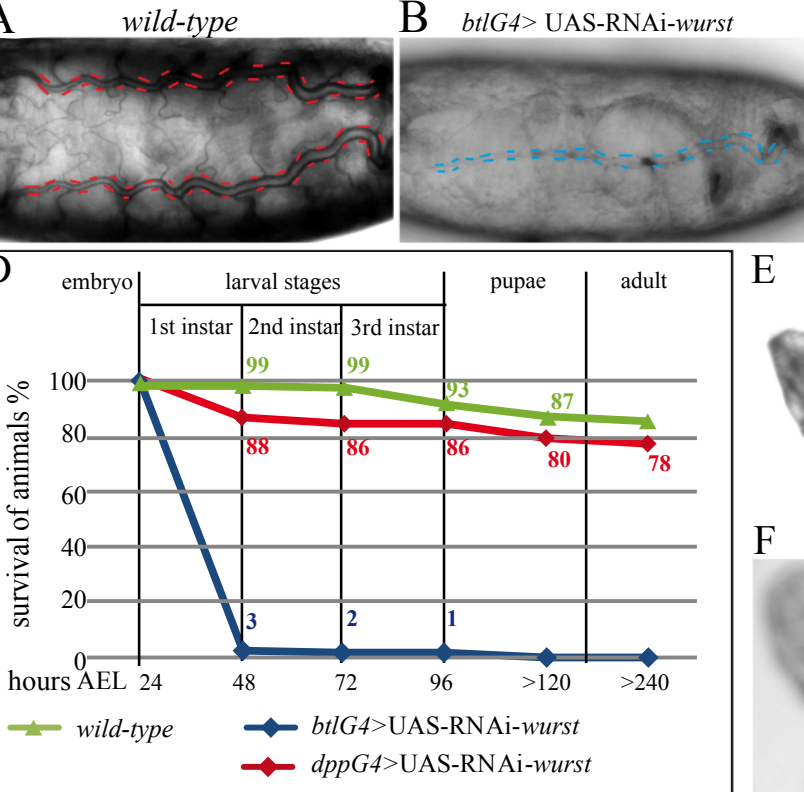

C $d p p G 4>$ UAS-RNAi-wurst

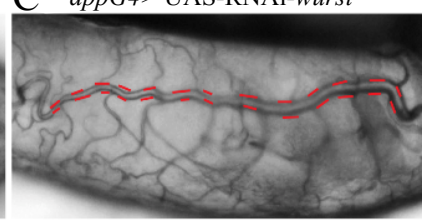

E
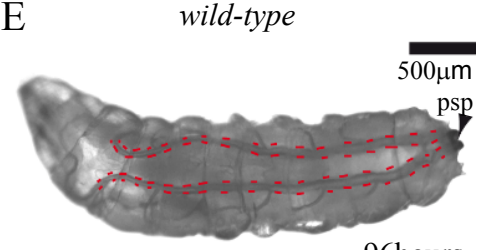

96hours

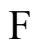

btlG4> UAS-wurstRNA

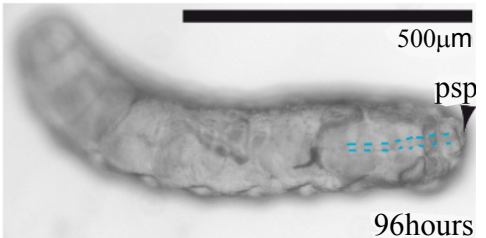

$500 \mu \mathrm{m}$

Figure 3: Wurst-mediated airway clearance is essential for developmental progress

Images show bright-field microscopy of living embryos at stage 17. Red dashes indicate air-filled, blue dashes liquid-filled airways. (A) Wild-type embryos undergo airway liquid-clearance and air-filling (B) Tracheal specific RNAi-mediated wurst knock-down resulted in severe air-filling defects (C) In contrast, posterior spiracles (respiratory openings) specific RNAi-mediated wurst knock-down did not lead to air-filling defects. (D) Histogram indicates survival in percentage of animals throughout development AEL. In contrast to wild-type, the tracheal specific RNAi-mediated wurst knock-down is lethal to early larvae. The RNAi-mediated wurst knock-down in posterior spiracles is not lethal, when compared to wild-type. Values in percentage are indicated in green, red and blue. Tested animals: wild-type $\mathrm{n}=300$; dppG4>UAS-wurst RNAi n=248; bt/G4>UAS-wurstRNAi n=492. (E,F) Images display wild-type (E) and tracheal specific wurstRNAi knock-down (F) larvae about 96 hours AEL. Black bars indicate $500 \mu \mathrm{m}$. Wild-type, which reaches the final larval stage, shows characteristic darkening and evagination of posterior spiracles. In contrast, the development of RNAi-wurst knock-down larvae is arrested. The body size is smaller, when compared to wild-type, and darkening of the posterior spiracles is not observed in the wurst knock-down mutants. 


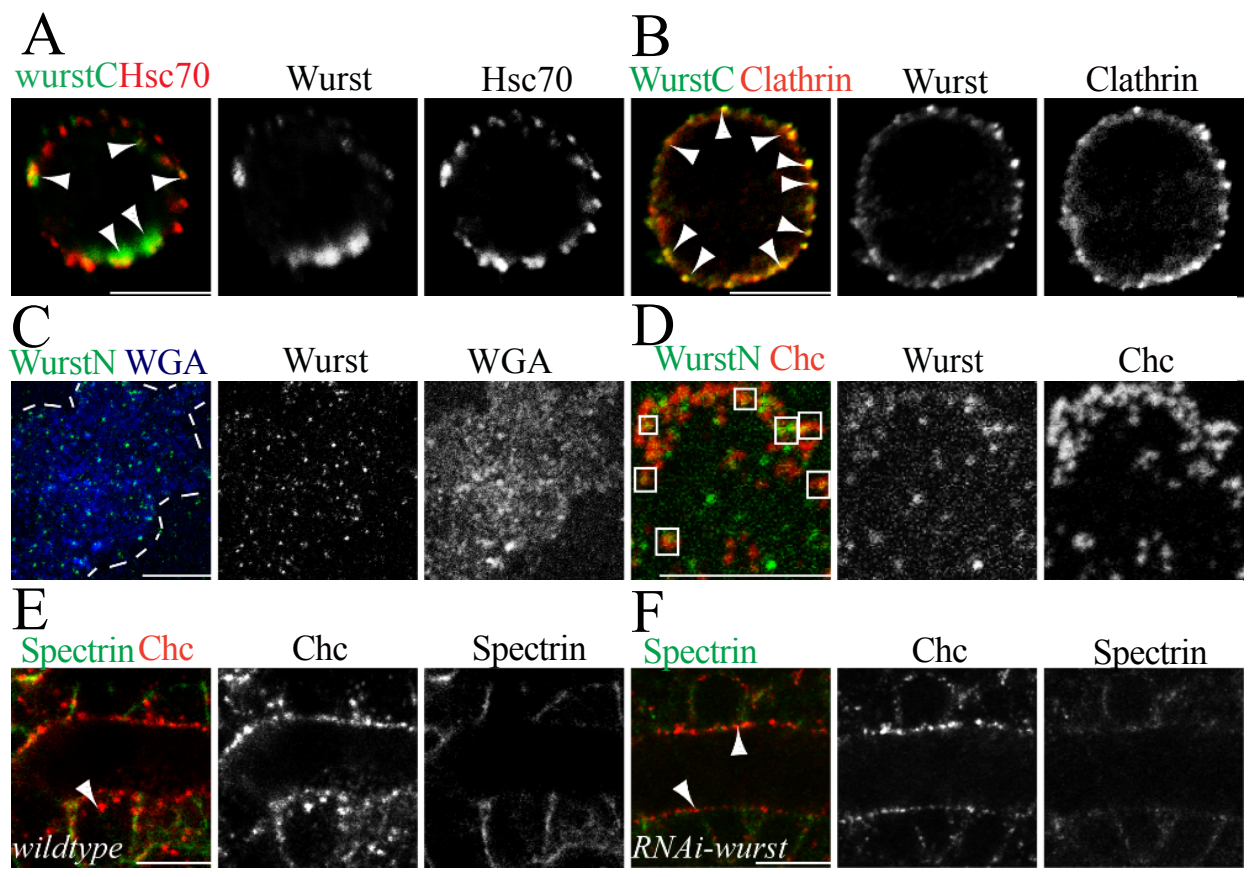

Figure 4: Wurst is involved in clathrin-mediated endocytosis

(A-D) Immunofluorescent analysis using anti-clathrin, anti-Chc, anti-Hsc70, anti-WurstC (recognizing the C-terminus [13]), and anti-WurstN (recognizing the N-terminus, see materials and methods) antibodies in Drosophila SL2 cells (A,B) and in membrane sheets of SL2 cells (C,D). The anti-clathrin antibody recognizes Chc and Clathrin light chain. $(A, B)$ Wurst is localized in a vesicular like pattern in SL2 cells, which partially co-localize (arrowheads) with Hsc70 (A) and clathrin (B). (C) Wurst appears distributed in small clusters in the membrane sheet of a SL2 cell. Wheat germ agglutinin (WGA) selectively recognizes sugar residues at the plasma membrane surface. White dashes mark the membrane sheet. (D) Magnification of the membrane sheet reveals Wurst (green) and Chc (red) overlap (rectangles). (E,F) Confocal images using anti-Chc and anti-a-Spectrin antibodies in Drosophila stage 17 embryos. The $\alpha$-Spectrin marks cell membranes. In wild-type tracheal cells Chc is found at the membrane and in addition in the cytoplasmic cell cortex (arrowhead in E). In contrast, in bt/G4 driven UAS-RNAi-wurst tracheal specific knock-down embryos, Chc is accumulated at the apical plasma membrane of tracheal cells (arrowheads in F). Scale bars indicate $5 \mu \mathrm{m}$.

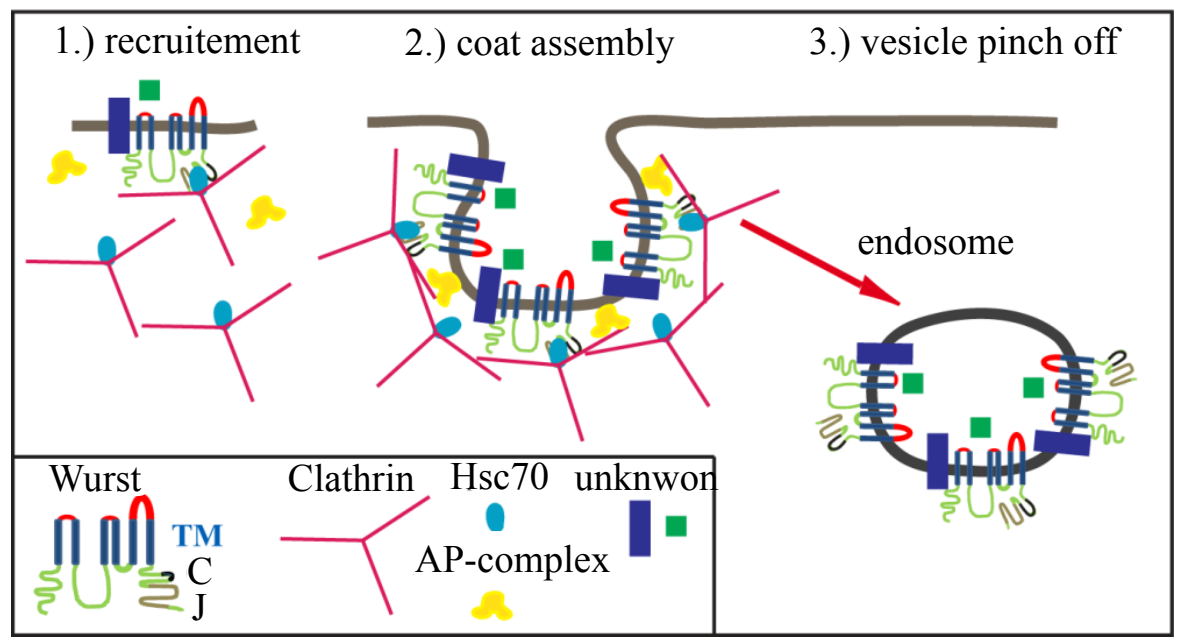

Figure 5: The evolutionarily conserved DnaJ-domain protein Wurst is involved in endocytosis

(A) The cartoon illustrates the putative role of Wurst in endocytosis of clathrin-coated vesicles. The predicted Wurst structure contains six transmembrane domains (TM: blue), a clathrin-binding motif (C; black) and a J-domain ( $\mathrm{J}$; grey).Wurst cytoplasmic parts are indicated in green, the extracellular in red. Clathrin assembles a coatlike structure around the nascent vesicle, aided by adaptor proteins (AP-complex). After vesicles scission, clathrin disassembles and can be reused for a new round of endocytosis. The uncoated vesicle pinches off and fuses with acceptor compartments such as early endosomes. Our data indicate that Wurst recruits clathrin and Hsc70-4 to the apical membrane. In contrast to clathrin and Hsc70-4, Wurst remains in the vesicle, which fuses to acceptor compartments, such as early endosomes. Thereby, Wurst may coordinate the endocytosis of unknown factors, which are involved in airway liquid-clearance.

Chc is predominantly localized at the apical plasma membrane as well as in the cortical cytoplasm (Figure $4 \mathrm{E}$ ). In contrast, the organ specific
Wurst knock-down in tracheal cells resulted in Chc accumulation only at the apical membrane (Figure $4 \mathrm{~F}$ ). In summary, these data identify 
Wurst as an essential component of the clathrin-mediated endocytosis in tracheal cells.

\section{Discussion}

Our results demonstrate the importance of Wurst in airway clearance and air-filling. Tissue specific rescue and RNAi-mediated Wurst knock-down experiments provided evidence that Wurst is specifically required in the differentiated tracheal cells and not in the spiracles as putative openings for air-filling at the end of embryogenesis. Moreover, the knock-down experiments showed the specific tracheal importance of Wurst function also for postembryonic air-filling, for developmental growth control and finally for survival. Furthermore colocalization assays in SL2 cells are in line with supposed Wurst function for clathrin-mediated endocytosis [13]. In addition, membrane sheet assays indicate that Wurst appears in clusters in the cell membrane and overlaps with Chc. Tissue specific knock-down experiments finally demonstrate the dependency of Chc on Wurst function in tracheal cells at the end of embryogenesis, when airway clearance occurs. We propose amodel (Figure 5) showing how Wurst may control the endocytosis of ECM matrix components for degradation thereby clearing the airway lumina at the end of embryogenesis. In addition, regarding its key function for airway liquid-clearance [14], we suggest that Wurst is involved in the endocytosis of channels, which are required for the liquid-clearance process. Epithelial Sodium Channels (ENaCs) are located at the apical membrane and establish a Sodium gradient, which regulates liquid absorption from airway lumina into the interstitium [5]. The activity of apical ENaCs is regulated by clathrin-mediated endocytosis [24]. Strikingly, apical Wurst localization in tracheal cells is essential for airway liquid-clearance [14]. Moreover, genetic studies demonstrate interaction of Wurst with Drosophila ENaCs [13], which are encoded by the pickpocket genes [25]. In summary, we assume that Wurst is required for regulating Drosophila ENaCs activity in order to control airway liquid-clearance. Wurst and its human ortholog DNAJC22 share conserved sequence and domain structure [15], suggesting that they could have similar function in clathrin-mediated endocytosis. Whether DNAJC22 plays a similar role in humans in endocytosis of $\mathrm{ENaC}$ is not yet known.

\section{Acknowledgement}

We are grateful to the Bloomington and Havard (Exelixis) stock centers for providing us with fly stocks. We thank Andreas Fink, Nataliya Nedzhvetskaya and members of the Hoch lab for critical reading of the manuscript. We thank Sabine Büttner and Nina Lülsdorf for technical assistance. We are very grateful to Thorsten Lang and Luis Spitta for help and discussions in generating membrane sheets. We appreciate the support provided by Michael Hoch. M.B. was supported by the FritzThyssen-Foundation (Az.10.08.2.138) and the Deutsche Forschungsgemeinschaft (SFB645)

\section{References}

1. Metzger RJ, Klein OD, Martin GR, Krasnow MA (2008) The branching programme of mouse lung development. Nature 453: 745-750.

2. Affolter M, Bellusci S, Itoh N, Shilo B, Thiery JP, et al. (2003) Tube or not tube: remodeling epithelial tissues by branching morphogenesis. Dev Cell 4: 11-18.

3. Affolter M, Zeller R, Caussinus E (2009) Tissue remodeling through branching morphogenesis. Nat Rev Mol Cell Biol 10: 831-842.

4. Olver RE, Walters DV, Wilson SM (2004) Developmental regulation of lung liquid transport. Annu Rev Physiol 66: 77-101.

5. Elias N, O'Brodovich H (2006) Clearance of Fluid From Airspaces of Newborns and Infants. Neo Reviews 7: e88-e94.

6. Birse RT, Bodmer R (2011) Lipotoxicity and cardiac dysfunction in mammals and Drosophila. Crit Rev Biochem Mol Biol 46: 376-385.
7. Crowther DC, Page R, Rival T, Chandraratna DS, Lomas DA (2008) Using a Drosophila model of Alzheimer's disease. SEB Exp Biol Ser 60: 57-77.

8. Horowitz A, Simons M (2009) Branching morphogenesis. Circ Res 104: e21.

9. Uv A, Cantera R, Samakovlis C (2003) Drosophila tracheal morphogenesis: intricate cellular solutions to basic plumbing problems. Trends Cell Biol 13: 301 309.

10. Schottenfeld J, Song Y, Ghabrial AS (2010) Tube continued: morphogenesis of the Drosophila tracheal system. Curr Opin Cell Biol 22: 633-639.

11. Affolter M, Caussinus E (2008) Tracheal branching morphogenesis in Drosophila: new insights into cell behaviour and organ architecture. Development 135: 2055-2064.

12. Behr M (2010) Molecular aspects of respiratory vascular tube development Respir Physiol \& Neurobiol 173: S33-36.

13. Behr M, Wingen C, Wolf C, Schuh R, Hoch M (2007) Wurst is essential for airway clearance and respiratory-tube size control. Nat Cell Biol 9: 847-853.

14. Stümpges B, Behr M (2011) Time-specific regulation of airway clearance by the Drosophila J-domain transmembrane protein Wurst. FEBS Lett 585: 3316 3321.

15. Wingen C, Aschenbrenner AC, Stumpges B, Hoch M, Behr M (2009) The Wurst protein: A novel endocytosis regulator involved in airway. Cell Adh Migr 3: 14-18.

16. Ungewickel EJ, Hinriches L (2007) Endocytosis: clathrin-mediated membrane budding. Curr Opin Cell Biol 19: 417-425.

17. Wingen C, Stümpges B, Hoch M, Behr M (2009) Expression and localization of clathrin heavy chain in Drosophila melanogaster. Gene Expr Patterns 9: 549-554.

18. Brand AH, Perrimon N (1993) Targeted gene expression as a means of altering cell fates and generating dominant phenotypes. Development 118: 401-415.

19. Takaesu NT, Johnson AN, Newfeld SJ (2002) Posterior spiracle specific GAL4 lines: new reagents for developmental biology and respiratory physiology. Genesis 34: 16-18.

20. Wingrove JA, O'Farrell PH (1999) Nitric oxide contributes to behavioral, cellular and developmental responses to low oxygen in Drosophila. Cell 98: 105-114.

21. Henry JR, Harrison JF (2004) Plastic and evolved responses of larval tracheae and mass to varying atmospheric oxygen content in Drosophila melanogaster. J Exp Biol 207: 3559-3567.

22. Lang T, Bruns D, Wenzel D, Riedel D, Holroyd P, et al. (2991) SNAREs are concentrated in cholesterol-dependent clusters that define docking and fusion sites for exocytosis. EMBO J 20: 2202-2213.

23. Sieber JJ, Willig KI, Kutzner C, Gerding-Reimers C, Harke B, et al. (2007) Anatomy and Dynamics of a Supramolecular Membrane Protein Cluster Science 317: 1072-1076.

24. Shimkets RA, Lifton RP, Canessa CM (1997) The activity of the epithelia sodium channel is regulated by clathrin-mediated endocytosis. J Biol Chem 272: 25537-25541.

25. Liu L, Johnson WA, Welsh MJ (2003) Drosophila DEG/ENaC pickpocket genes are expressed in the tracheal system, where they may be involved in liquid clearance. Proc Natl Acad Sci USA 100: 2128-2133.

This article was originally published in a special issue, Pulmonary developmental biology handled by Editor(s). Dr. Rodolfo de Paula Vieira, University Hospital Freiburg, German 